\title{
Towards Realism in an Intelligent Car's Driving Behavior Using Fuzz Inference System
}

\author{
Nima Fouladinejad, Nariman Fouladinejad, J. Mohd Taib, and M. K. Abd Jalil
}

\begin{abstract}
This paper will discuss the algorithm to be implemented in the simulation of traffic flow system with the presence of the intelligent vehicles inside a microscopic traffic flow. This is possible with the introduction of the Behavior Sector to set various driving behavior of the virtual intelligent vehicles. The Behavior Sector consists of five different units, which are Route and Destination, Vision, Personality, Decision Making, and Motion Control. Fuzzy Logic is adopted to control the Behavior Sector. The traffic flow system is developed using Matlab software using Fuzzy Logic toolbox. One case study based on Taman Universiti area in Skudai, Johor is used to show the results of the Behavior Sector in the simulation of the traffic flow system.
\end{abstract}

Index Terms-Microscopic traffic flow model, intelligent vehicle, fuzzy inference system (FIS), realistic decision module.

\section{INTRODUCTION}

With the advance of computing and complex techniques in programming, the simulation of a real driver behavior inside the virtual environment is possible. The foundation requirement is to develop a framework to mimic what a real driver does while driving. The framework to imitate human driving behavior requires a number of factors to be taken into consideration [1]. Amongst the factors that have influences on the behavior considered in this research are destination, vision, personality, decision, and controlling of motion.

This research's framework composes five units and each of them handles certain attribute in mimicking a real driver behavior in driving.

These five units are the Route and Destination Unit, Vision Unit, Personality, Mental and Physical Condition Unit, Decision Making Unit and Motion Control Unit. The link between the units is shown in Fig. 1. The Route and Destination Unit encompasses all the other units. In the case of normal driving, the behavior will follow all the five units in sequence, however when in the case of emergency detected by the vision system, the framework will go straight to motion control unit without having to go through Decision Making Unit.

Unlike most of the previous researches and articles in the field of intelligent traffic system which adopted expert system like Micro-simulation of road traffic [2] or Traffic Light Simulations [3], this article attempts to minimize the gap of being unrealistic in traffic simulation by using FIS,

Manuscript received February 20, 2014; revised May 19, 2014. This work was supported by Ministry of Education of Malaysia and UTM Research Management Center.

Nima Fouladinejad, Nariman Fouladinejad, J. Mohd Taib, and M. K. Abd Jalil are with Faculty of Mechanical Engineering, Universiti Teknologi Malaysia, Malaysia (e-mail: nima.fouladinejad@gmail.com, nariman.fouladinejad@gmail.com). which has been used broadly in engineering applications in the recent years [4]. Fuzzy as the mean for the data-fusion have been mostly used as a tool to fuse the data retrieved from multisensor [5], [6] or more recently it is used in fusing position signals from Global Positioning Systems (GPS) and inertial navigation systems (INS) for autonomous mobile vehicles [7]. Moreover, the potential of applying fuzzy logic techniques in traffic and transportation systems analysis and control are also been discussed in few articles [8], [9].

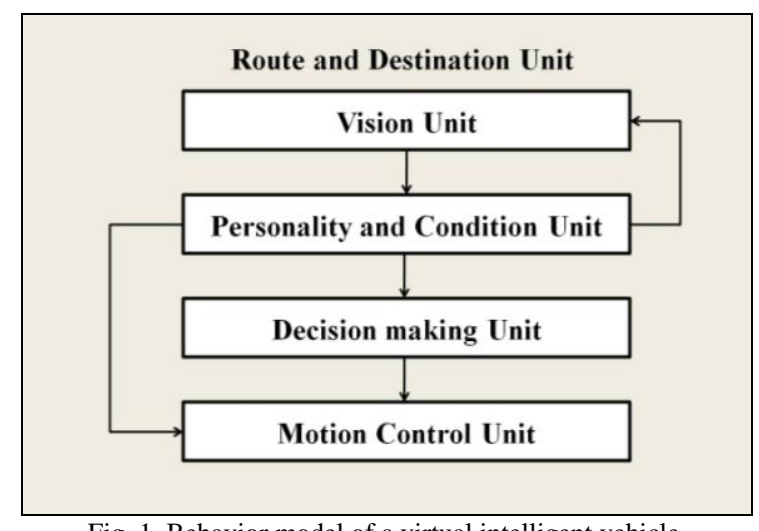

Fig. 1. Behavior model of a virtual intelligent vehicle.

In this research, fuzzy system will fuse the input data from various sources into more realistic definitions that will set the driving behaviors of the intelligent vehicles. In the following, it will discuss the implementation of the fuzzy system in the development of the system.

\section{Route AND DESTINATION UNIT}

This unit will be infolded in an M-File in the main program. This M-File will draw the route for each intelligent agent at the beginning of the program by taking into account the starting position and the final destination of each intelligent car. After assigning the route to each intelligent car, the intelligent cars will receive the data from the overall database, which consists of all the data from the other cars and surroundings, within their specified routes. This method will make the programming techniques more efficient, in addition to make the driving behavior more realistic, especially in a city map simulation with all those junctions and traffic lights.

\section{VISION UNIT}

This unit is responsible in receiving information from surrounding and other vehicles inside the simulation environment, and using them in the decision making in any driving situation. To make this unit more realistic to human 
vision, the range of vision can be influenced by different factors like drugs, drinks, illness or exhaustion. This is done by the introduction of a Visibility Circle. The range of visibility depends on the type of the drivers assigned to the intelligent cars. Fig. 2 demonstrates the range of the visibility circle.

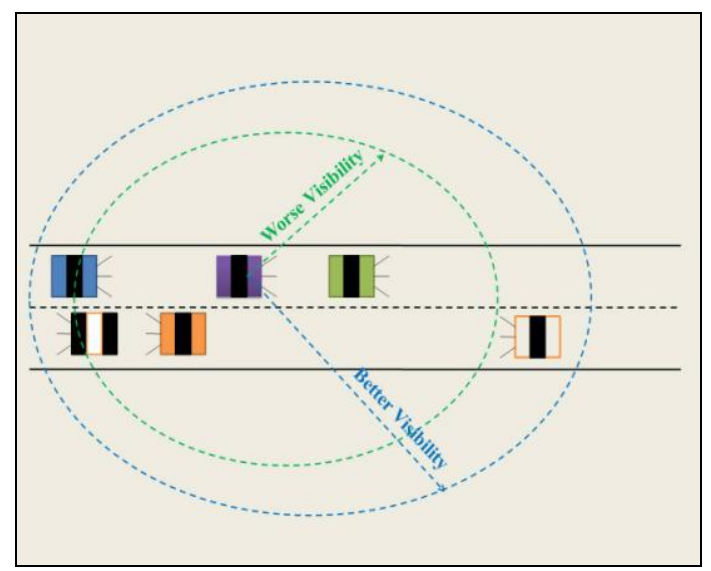

Fig. 2. Definition of visibility circle.

The method that is used in this research for each intelligent car to "See" in the virtual environment is Overall Database Technique [10]. In this technique, each intelligent car will get access to an overall database (in their assigned route regarding the previous section and also inside their visibility circle) and they will receive data regarding the position of other cars, surroundings and obstacles in the virtual environment. The unique feature in this technique is that, the data that is received by each intelligent car will be fused to some realistic definitions, using Fuzzy logic system.

These fused data will make the vision section more realistic, just like a real driver. For instance, in reality, the driver will only judge the distance between his/her car with the car in front. Instead, he/she will judge the distance in the format of some definitions like; near, so near, or far. As shown in Fig. 3, Fuzzy Logic will define the raw data based on the distance, into some intervals and it will be fuzzified in order to generate the fused data. These fused data will allow the simulation to receive the distances for each intelligent car, in similar manner with a real driver, driving in an actual driving experience.

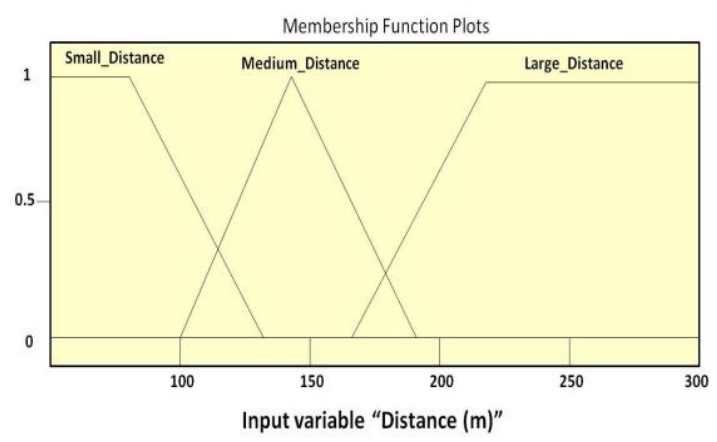

Fig. 3. Membership function for fuzzifying the distance.

\section{Personality, Mental and Physical Condition UNIT}

This unit contains all the characteristics, moods or emotions of the driver. These factors can affect some of the driving preferences like; choice of speed, distance to other cars, rate of acceleration and deceleration, etc. Like previous unit, to make the unit more realistic, all of the preferences can be influenced by external factors, such as age, drugs, drinks, illness or exhaustion. The technique, that is utilized in this research to reflect the "Personality, Mental and Physical Condition Unit" in the MATLAB programming, is called as Fuzzy Interference System. The method will use series of Fuzzy Rules to establish the personality or driving preference. Some samples of the Fuzzy rules are shown in Fig. 4.

These Fuzzy Rules will access the overall database to get all the required input data, and produce the output to the next unit. It is like writing a driving personality for a driver as some Fuzzy Rule, and then expects the outcome-behavior, by the driver, in every driving situation.

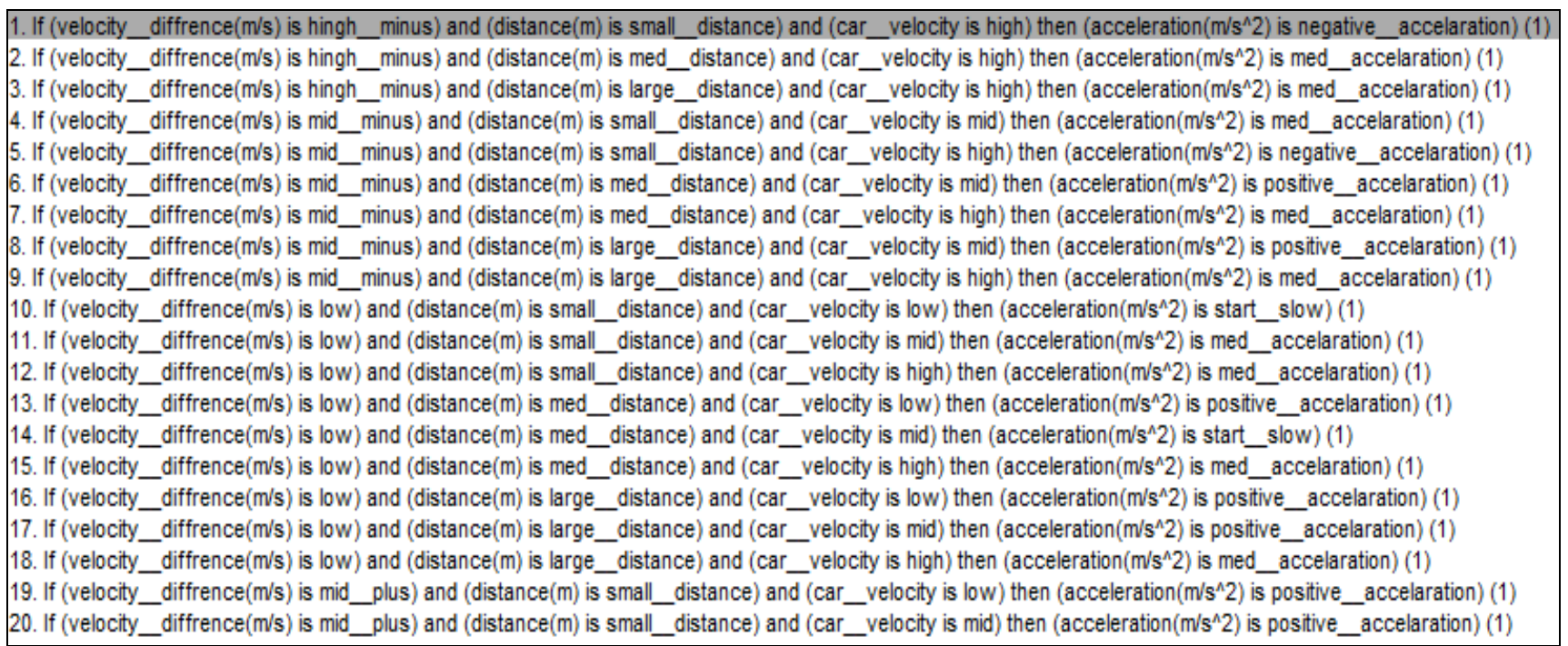

Fig. 4. Fuzzy Rules, generating personality unit.

It is worth mentioning that, all the inputs that are used in this section by fuzzy rules are represented in a realistic way, similar to the vision unit. Each input is categorized into some intervals in order to make the Decision Making System 
becomes more realistic. This is similar to the real driving experience, when the driver, for instance, cannot guess the exact speed difference between his/her car with the car in front. Instead, he/she will have some definitions for each state of difference in velocity with the car in front, like real fast or slow. By getting access to these inputs from the environment and other cars and converts them into more realistic definitions, the driving preference will be established and this driving preference becomes the input to the Decision Making Unit.

\section{DECISION MAKING UNIT}

This unit is responsible in making the appropriate decision based on the driving preference from the previous unit. For example, in an emergency braking situation, driver with low level of vision will take longer time to react. The time taken to make decision differs from one driver to another. As previously mentioned, after accessing the overall database acted as the vision unit, and also getting access to all the Fuzzy Rules, that represents the Personality of the driver, the next step is to generate some outputs as the Decision in every driving situations. It can be said that FIS, which is "Mamdani" type for this research, is responsible of making the best decision regarding all the inputs and rules that are defined for the system. Fig. 5 illustrates the FIS type and format that is used for this research. The Fuzzification motor will get access to all the seven inputs data and with regards to a related part of the fuzzy rules that are written by the user, it will generate some outputs. These outputs can be called as driving decisions and they will be used by the Motion Control Unit later.

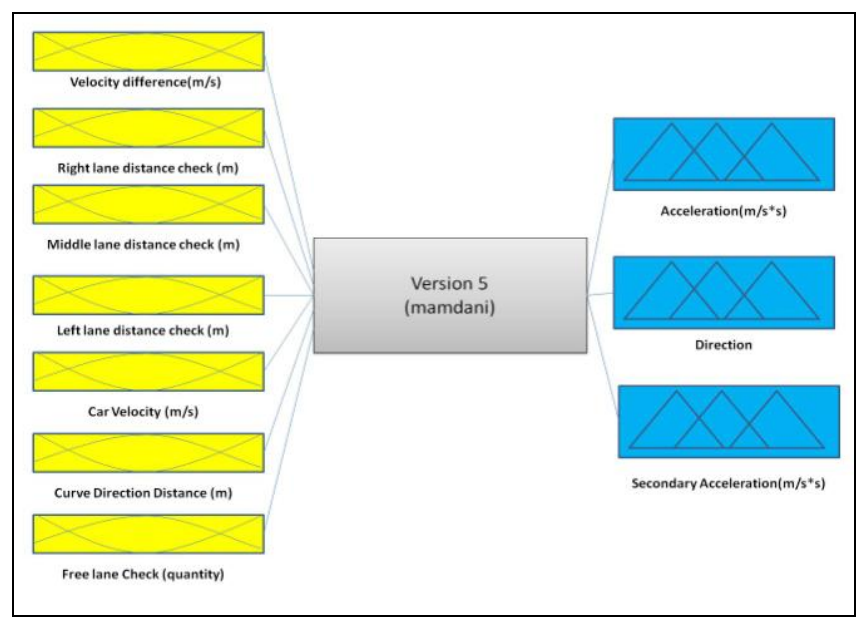

Fig. 5. FIS Format for this research (Mamdani type).

The inputs to the fuzzification machine are: velocity difference (between the leader and follower car), right lane distance check (to observe obstacle in the right lane), middle lane distance check (to observe obstacle in the middle lane), left lane distance check (to observe obstacle in the left lane), car velocity, curve direction and distance (the next turn according to the assign route), and free lane quantity Check (the number of empty lane in right and left side, which is used for lane change decision). Furthermore, there are three different types of outputs, which will govern the reaction on the accelerator pedal, brake pedal and steering, and they are: main acceleration, direction and secondary acceleration.

\section{MOTION CONTROL UNIT}

This section is responsible to translate the decision into motion. To make the action programmable, mathematical form of driving maneuvering is used. With the mathematical form, the maneuvering path for the intelligent car can be generated. The mathematical form is generated by the integration of the Hermite Curve and Dynamic of Motion. This mathematical form has been discussed in our previous research [11]. The transformation process relies on the decision-time taken for each driver. Finally, the motion of the vehicle can be simulated in the system with some $2 \mathrm{D}$ images.

\section{CONCLUSION}

This platform is able to support different types of drivers like aggressive, old, young, nearsighted, farsighted, and even to simulate drunk and drugged drivers, by changing the fuzzy input intervals and modifying the Fuzzy Rules. The most observable advantage of this research compare to other researches, in the same field, is being realistic and flexible. Needless to say, there are far more many things in the realm of autonomous vehicle that have not been covered in this study. Furthermore, in the future work beyond the content of this research can use Neural Network to allow the Intelligent Agent to learn from its driving faults and to correct them in subsequent similar situations.

\section{ACKNOWLEDGMENT}

The authors would like to thank Ministry of Education and UTM Research Management Center for providing grants to carry out the research.

\section{REFERENCES}

[1] T. Al-Shihabi and R. R. Mourant "A framework for modeling human-like driving behaviors for autonomous vehicles in driving simulators," in Proc. the 5th International Conf. on Autonomous Agents, Montreal, Canada, May 2001, pp. 286-291.

[2] M. Treiber, Micro-simulation of Road Traffic, Institute for Transports and Economics, Dresden University of Technology, 2008.

[3] K. Deibel, Traffic Light Simulations, Computer Science \& Engineering, University of Washington, 2007.

[4] T. J. Ross, "Fuzzy logic with engineering applications," McGraw-Hill, New York, 1995.

[5] F. Russo and G. Ramponi, "Fuzzy methods for multisensor data fusion," IEEE Trans. Instrum. Meas., vol. 43, no. 2, pp. 288-294, 1994.

[6] J. A. Stover, D. L. Hall, and R. E. Gibson, "A fuzzy-logic architecture for autonomous multisensor data fusion," IEEE Trans. Ind. Electron., vol. 43, 1996.

[7] J. Z. Sasiadek, Q. Wang, and M. B. Zeremba, "Fuzzy adaptive Kalman filtering for INS/GPS data fusion," in Proc. 15th IEEE Int. Symp. Intell. Control, 2000, pp. 181-186.

[8] S. Hoogendoorn, S. Hoogendoorn-Lanser, and H. Schuurman, "Fuzzy perspectives in traffic engineering," Research Report, TRAIL Research School, Delft, Report on Behalf of Dutch Ministry of Transport, 1998.

[9] U. Chattaraj and M. Panda, "Some applications of fuzzy logic in transportation engineering," in Proc. International Conference on Challenges and Applications of Mathematics in Science and Technology (CAMIST), NIT Rourkela, 2010.

[10] H. W. He, Y, M. Lu, and Y. Lou Yan, "Virtual reality based intelligent vehicle modeling in driving SIMULATION system," in Proc. 7th International Conference on Computer-Aided Industrial Design and Conceptual Design, pp. 1-5, 2006. 
[11] N. Foulidinejad, N. Foulidinejad, J. M. Taib, and M. K. A. Jalil, "Intelligent traffic flow simulation system on a small scale environment," in Proc. $4^{\text {th }}$ International Conference on Computer and Electrical Engineering, Singapore, October 14-15, 2011, pp. 27-32.

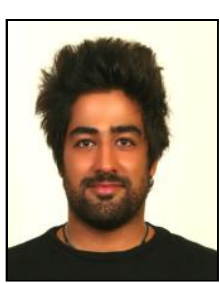

Nima Fouladinejad received his B.Sc. degree in mechanical engineering in the field of manufacturing and production from Najaf Abad University of Esfahan, Iran in 2008. He did his M.Sc. degree in the field of design in mechanical engineering at Universiti Teknologi Malaysia in 2010. He is now a final-year $\mathrm{PhD}$ student and he is working in the field of intelligent automobile and intelligent traffic system.

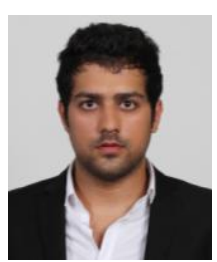

Nariman Fouladinejad received his B.Sc. degree in mechanical engineering in the field of manufacturing and production from Najaf Abad University of Esfahan, Iran in 2008. He did his M.Sc. degree in the field of design in mechanical engineering at Universiti Teknologi Malaysia in 2010. He is now a final year PhD student and he is working in the field of optimization of driving simulator subsystems.

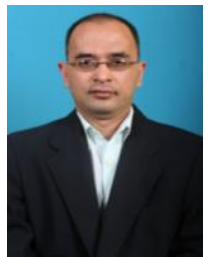

Jamaludin Mohd Taib is a senior lecturer at the Department of Applied Mechanic and Design, Faculty of Mechanical Engineering, Universiti Teknologi Malaysia. He did his B.Sc degree in mechanical engineering at The University of Texas at El Paso, Texas in 1988. He received his M. Phil degree in computer aided engineering at Heriot-Watt University of Scotland in 1992. He pursued his PhD degree in computer aided engineering at University of Dundee of Scotland in Year 1999. Currently his research fields are feature recognition (integration of feature recognition with future based modeling system), geometric reasoning and traffic flow model (TFM)

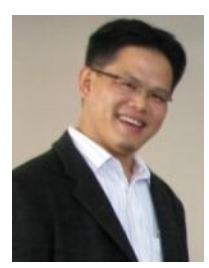

Mohamad Kasim bin Abdul Jalil is an associate professor at the Department of Applied Mechanic and Design in the Faculty of Mechanical Engineering, Universiti Teknologi Malaysia. He did his B.Sc degree in mechanical engineering at Union College of New York in 1989. He received his M.Sc. degree in engineering design at Loughborough University of Technology of England in 1993. He pursued his PhD degree in mechanical engineering (CAD/virtual reality) at The University of Buffalo, New York in 2003. Currently his research fields are computer-aided design, virtual reality applications in engineering, collaborative virtual environment, and cluster/parallel computing. 